\title{
Divergent effects of resistance and endurance exercise on plasma bile acids, FGF19, and FGF21 in humans
}

\author{
Thomas Morville, ${ }^{1}$ Ronni E. Sahl, ${ }^{1,2}$ Samuel A.J. Trammell, ${ }^{2}$ Jens S. Svenningsen, ${ }^{2}$ \\ Matthew P. Gillum, ${ }^{2}$ Jørn W. Helge, ${ }^{1}$ and Christoffer Clemmensen ${ }^{2}$ \\ ${ }^{1}$ Xlab, Center for Healthy Aging, Department of Biomedical Sciences, and ${ }^{2}$ Novo Nordisk Foundation Center for Basic \\ Metabolic Research, Faculty of Health and Medical Sciences, University of Copenhagen, Copenhagen, Denmark.
}

BACKGROUND. Exercise has profound pleiotropic health benefits, yet the underlying mechanisms remain incompletely understood. Endocrine FGF21, bile acids (BAs), and BA-induced FGF19 have emerged as metabolic signaling molecules. Here, we investigated if dissimilar modes of exercise, resistance exercise (RE) and endurance exercise (EE), regulate plasma BAs, FGF19, and FGF21 in humans.

METHODS. Ten healthy, moderately trained males were enrolled in a randomized crossover study of 1 hour of bicycling at $70 \%$ of $\mathrm{VO}_{2 \text { peak }}(\mathrm{EE})$ and 1 hour of high-volume $\mathrm{RE}$. Hormones and metabolites were measured in venous blood and sampled before and after exercise and at 15, 30, 60, 90, 120, and 180 minutes after exercise.

RESULTS. We observed exercise mode-specific changes in plasma concentrations of FCF19 and FGF21. Whereas FGF19 decreased following RE $(\boldsymbol{P}<0.001)$, FGF21 increased in response to EE ( $P$ $<0.001)$. Total plasma BAs decreased exclusively following $R E(P<0.05)$, but the composition of BAs changed in response to both types of exercise. Notably, circulating levels of the potent TCR5 receptor agonist, lithocholic acid, increased with both types of exercise $(P<0.001)$.

CONCLUSION. This study reveals divergent effects of EE and RE on circulating concentrations of the BA species, FGF19, and FGF21. We identify temporal relationships between decreased BA and FGF19 following RE and a sharp disparity in FGF21 concentrations, with EE eliciting a clear increase parallel to that of glucagon.

FUNDING. The Novo Nordisk Foundation (NNF170C0026114) and the Lundbeck Foundation (R2382016-2859).

Conflict of interest: The authors have declared that no conflict of interest exists.

Submitted: June 6, 2018 Accepted: June 21, 2018 Published: August 9, 2018

\section{Reference information:} JCI Insight. 2018;3(15):e122737. https://doi.org/10.1172/jici. insight.122737.

\section{Introduction}

Exercise has pleiotropic health benefits, and understanding the mechanisms by which exercise regulates human biology might advance better exercise protocols for disease prevention and could give rise to novel exercise-mimicking pharmacotherapies (1). Endocrine signals are upstream of a wide range of exercise-governed molecular and physiological adaptations and are profoundly determined by the mode of exercise. Our understanding, however, of how exercise-induced secreted factors promote long-term systemic health benefits is incomplete.

The bile acid (BA)/FGF19 axis is an endocrine feedback system of the enterohepatic circulation and crucial to the absorption of dietary lipids (2). Over the past decade the BA/FGF19 axis has emerged as a relevant endocrine axis in energy metabolism. Serum BAs contribute to the metabolic benefits of bariatric surgery and through activation of the membrane receptor TGR5, BAs increase energy expenditure and improve lipid and glucose metabolism (3-5). FGF19 is an endocrine FGF that is expressed upon BA-induced farnesoid X receptor (FXR) activation in the small intestine $(6,7)$. In addition to suppressing hepatic BA synthesis, FGF19 also influences energy balance and glucose metabolism via FGF receptors in metabolic target tissues, including liver, adipose tissue, and the brain, and is an important endocrine signal for metabolic health (8). 


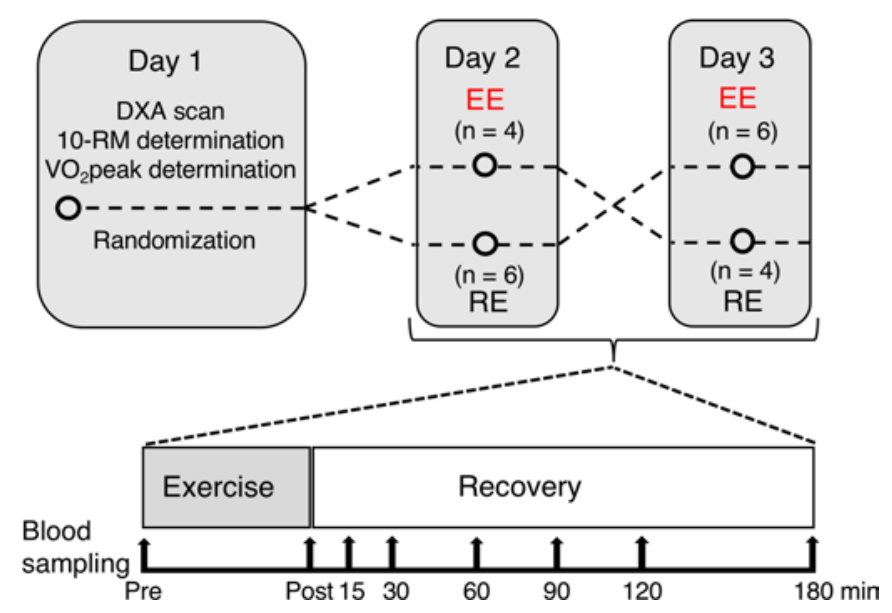

Figure 1. Study design. After randomization, all subjects performed both modes of exercise in a crossover design, separated by $8 \pm 2$ days. Exercise was performed for 60 minutes after an overnight fast. Blood sampling was performed before (Pre) and after (Post) exercise as well as during 3-hour recovery at the 15-, 30-, 60-, 90-, 120-, and 180-minute time intervals after exercise. $\mathrm{RM}$, repetition maximum; $\mathrm{EE}$, endurance exercise; $\mathrm{RE}$, resistance exercise.

Recently, pharmacological FGF19 administration was reported to induce skeletal muscle hypertrophy in mice, indicating a therapeutic potential for FGF19 in preventing atrophy-related complications, such as sarcopenia and cachexia (9). This feature is unique to FGF19, as pharmacological administration of FGF21, another member of the endocrine FGF family, has no direct effect on muscle size or strength in mice (9). Conversely, plasma FGF21 is reported to be increased with endurance exercise (EE) and may thus contribute to the benefits of this type of exercise on glucose and lipid metabolism (10-14). However, the temporal resolution of circulating FGF21 in response to EE, as well as the effect of resistance exercise (RE) on FGF21, has not yet been determined. Moreover, it remains to be investigated if the BA/FGF19 axis is regulated by exercise.

In the present work, a controlled randomized crossover study (Figure 1) was used to determine if exercise regulates circulating BA species, FGF19, and FGF21 in young healthy human male subjects and to unravel possible exercise mode-specific alterations. Because of the interconnectedness among BA, the endocrine FGFs, and canonical substrate-regulating hormones, we also profiled circulating insulin and glucagon in response to $\mathrm{EE}$ and RE.

\section{Results}

Diverging effects of EE and RE on circulating blood glucose, lactate and plasma insulin and glucagon concentrations in young healthy men

Blood glucose concentration was increased after RE and remained elevated at the 30-minute time point compared with $\mathrm{EE}$, with the largest difference at the 15 -minute time point: $103 \pm 6 \mathrm{mg} / \mathrm{dl}$ after RE and 85 $\pm 3 \mathrm{mg} / \mathrm{dl}$ after EE $(P<0.001$; Figure 2A). Blood glucose concentration was unchanged after EE.

Blood lactate concentration was increased 18-fold immediately after RE and 7-fold after EE. The increase after RE was significantly higher than that after EE, and the blood lactate concentration remained elevated during the first 90 minutes of recovery $(P<0.001$ for postexercise, $15-, 30-$, and 60-minute time points; $P=$ 0.036 for the 90 -minute time point; Figure $2 \mathrm{~B}$ ).

The plasma insulin concentration was $41.1 \pm 4.2 \mathrm{pg} / \mathrm{ml}$ before exercise, and it decreased to $19.9 \pm$ $7.2 \mathrm{pmol} / 1$ following EE. In contrast, the plasma insulin concentration for RE increased from $41.7 \pm 4.8$ $\mathrm{pmol} / 1$ before exercise to $54.4 \pm 7.6 \mathrm{pmol} / 1$ immediately after exercise. Plasma insulin concentration followed the same pattern as blood glucose and was elevated during the first 30 minutes after RE compared with $\mathrm{EE}(P<0.001$ for postexercise and 15 - and 30-minute time points; Figure $2 \mathrm{C})$.

Plasma glucagon concentration increased from $25.7 \pm 3.3 \mathrm{pg} / \mathrm{ml}$ before exercise to $72.5 \pm 7.5 \mathrm{pg} / \mathrm{ml}$ after EE, whereas plasma concentration was $32.0 \pm 4.4 \mathrm{pg} / \mathrm{ml}$ before exercise and was unchanged after RE $(40.6 \pm 6.8 \mathrm{pg} / \mathrm{ml})$. A statistical difference between modes of exercise was present at the postexercise and 15 and 30-minute time points $(P<0.001$; Figure 2D). The numerical, nonsignificant difference was consistent throughout the recovery; a significant difference was again apparent at the 90 -minute time point $(P=0.007)$. 

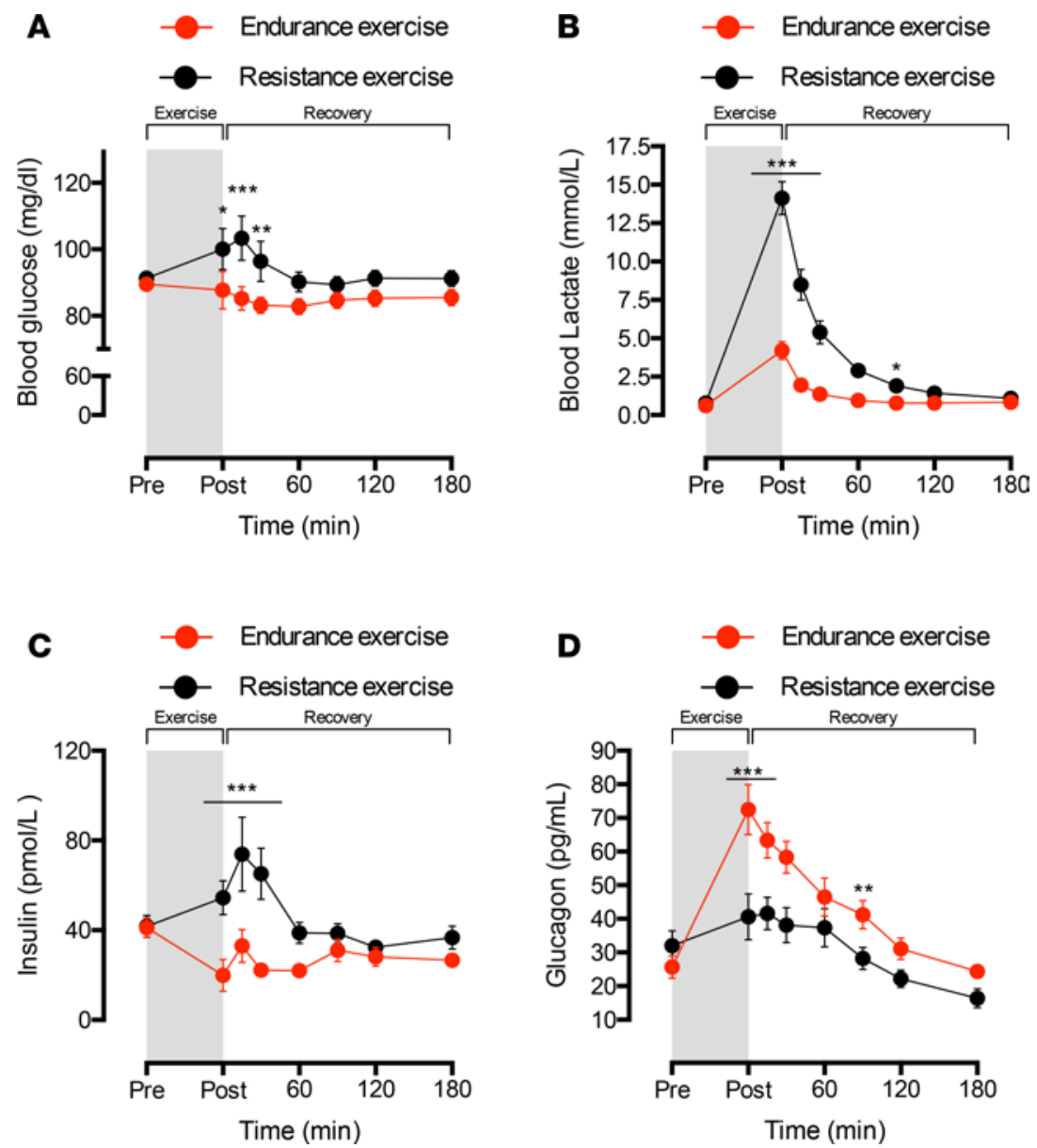

Figure 2. Exercise mode-dependent effects on plasma glucose, lactate, insulin, and glucagon. Effect of endurance exercise (red, $n=10$ ) and resistance exercise (black, $n=10$ ) on concentrations of blood glucose (A), blood lactate (B), plasma insulin (C), and plasma glucagon (D). Data are presented as mean $\pm \mathrm{SEM}$. ${ }^{*} P<0.05,{ }^{*} P<0.01,{ }^{* *} P<0.001$, comparing modes of exercise by 2-way ANOVA with Bonferroni post hoc test for multiple comparisons.

\section{Distinct effects of EE and RE on plasma FGF19 and FGF21}

Fasted FGF19 concentrations in plasma were $128 \pm 20$ and $155 \pm 38 \mathrm{pg} / \mathrm{ml}$ for EE and RE, respectively. FGF19 was not affected by EE and remained unchanged in the 3-hour recovery period (Figure 3A). Conversely, 90 minutes into the recovery period following RE, FGF19 was significantly lowered to $89 \pm 18$ $\mathrm{pg} / \mathrm{ml}$ compared with the concentration before exercise $(P=0.019)$, and this continued throughout the recovery phase to $60 \pm 9 \mathrm{pg} / \mathrm{ml}(P<0.001$ at 3 hours after exercise relative to that before exercise; Figure $3 \mathrm{~B})$. However, the decrease in plasma FGF19 concentration during the recovery phase after RE was not statistically different compared to that of EE (Figure 3C).

Plasma FGF21 concentrations before exercise were $46 \pm 11$ and $62 \pm 17 \mathrm{pg} / \mathrm{ml}$ for EE and RE, respectively. FGF21 increased by EE 15 minutes into the recovery $(74 \pm 20 \mathrm{pg} / \mathrm{ml})$, where it peaked at 60 minutes $(166 \pm 32 \mathrm{pg} / \mathrm{ml})$ and remained elevated until 120 minutes after exercise $(P<0.01$ for 15 -minute time point; $P<0.001$ for 30-, 60-, and 90-minute time points; Figure 3D). RE did not affect plasma FGF21 concentration at any time point (Figure 3E). Thus, plasma FGF21 concentration was higher from 30 to 120 minutes after EE compared with RE $(P<0.001$ for 30, 60, and 90 minutes after exercise and $P=0.023$ for 120 minutes after exercise; Figure $3 \mathrm{~F}$ ).

\section{Effects of EE and RE on plasma BA composition}

There was an effect of time $(P=0.04)$ on total BA (TBA) plasma concentration, primarily driven by a reduction in TBA following RE at 60 and 180 minutes after exercise compared with concentrations before exercise (from $2.37 \pm 0.57 \mu \mathrm{M}$ to $1.54 \pm 0.38$ and $1.40 \pm 0.34 \mu \mathrm{M}$, respectively; $P=0.03$ and $P=0.01$; 


\section{Endurance}

FGF-19

A

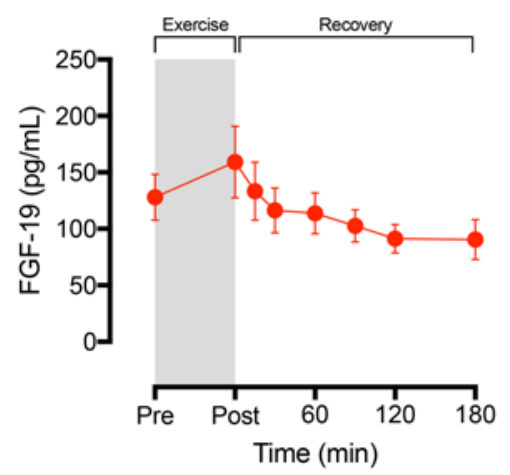

Resistance

B



Endurance vs. Resistance

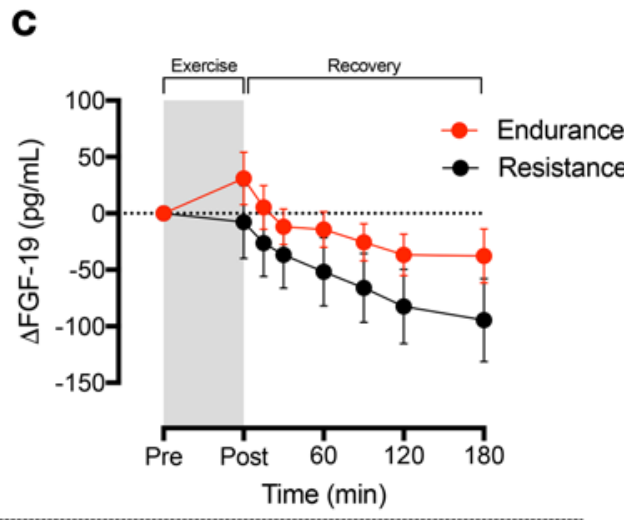

FGF-21

D

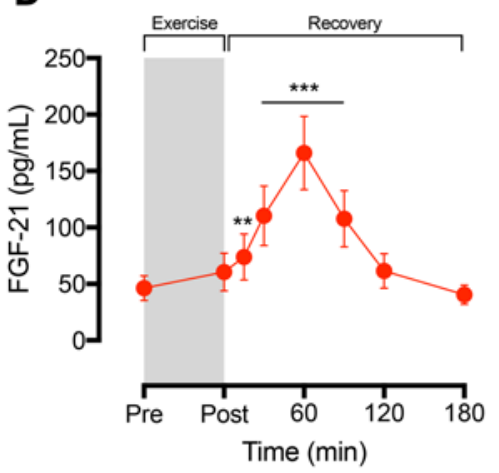

E

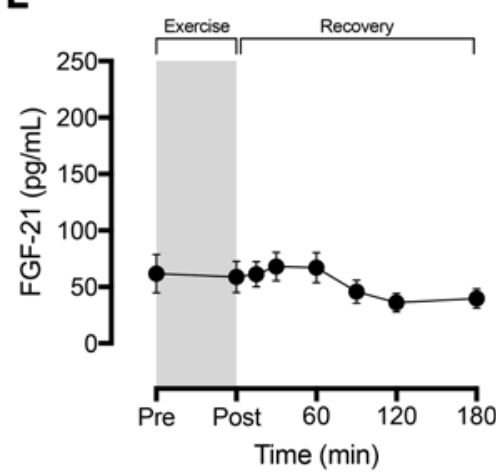

F



TBA

G

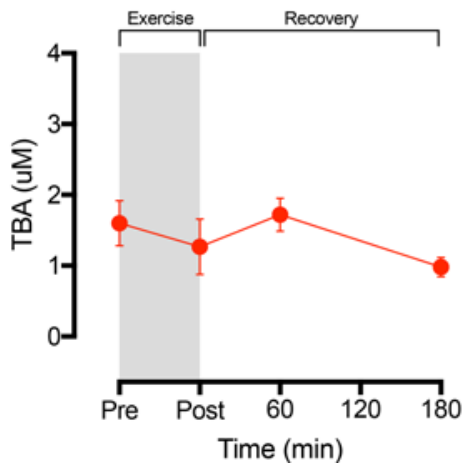

H



I

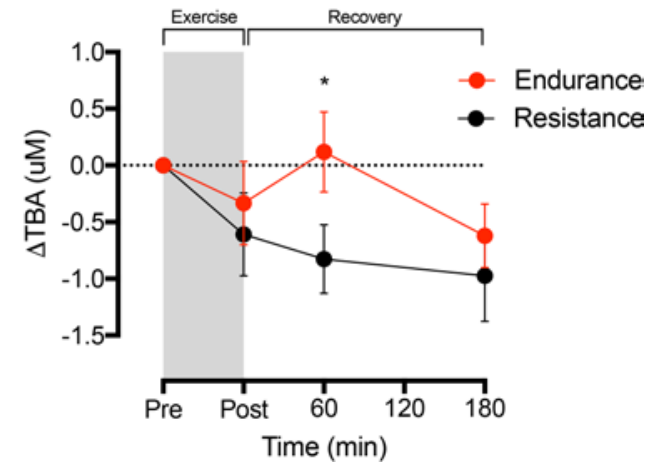

Figure 3. Effect of exercise on FGF19, FGF21, and total bile acids. Effects of endurance exercise (red) and resistance exercise (black) are shown for FGF19 ( $\mathbf{A}$ and B, $n=10$ for each exercise mode), FGF21 ( $\mathbf{D}$ and $\mathbf{E}, n=8$ for each exercise mode), and total bile acids (TBA) ( $\mathbf{C}$ and $\mathbf{H}, n=10$ for each exercise mode). $\Delta$ Changes from concentrations before exercise for both modes of exercise are shown for FGF19 (C), FGF21 (F), and TBA (I). Data are presented as mean \pm SEM. ${ }^{*} P<0.05$, ${ }^{* *} P<0.01$, ${ }^{* *} P<0.001$, comparing time points (difference from before exercise) by 2 -way ANOVA with Dunnett post hoc test for multiple comparisons. Differences between modes of exercise for each time point are shown by 2-way ANOVA with Bonferroni post hoc test for multiple comparisons in $\mathbf{C}, \mathbf{F}$, and $\mathbf{I}$ ).

Figure $3 \mathrm{H})$. There was no change in the TBA plasma concentration following EE, and RE was lower than $\mathrm{EE}$ at the 60 -minute time point after exercise $(P=0.02$; Figure $3, \mathrm{G}$ and $\mathrm{I})$. The individual BA concentrations are shown in Table 1, and an overview of the exercise-dependent regulation of plasma BA composition is shown in Figure 4. 
Table 1. Bile acid concentrations ( $\mu \mathrm{mol} / \mathrm{I})$ before and after EE and RE

\begin{tabular}{|c|c|c|c|c|c|c|c|c|c|c|c|}
\hline & \multicolumn{4}{|c|}{ Time points EE } & \multicolumn{4}{|c|}{ Time points RE } & \multicolumn{3}{|c|}{ Statistics } \\
\hline TBA & $1.60 \pm 0.32$ & $1.27 \pm 0.39$ & $1.72 \pm 0.23^{\mathrm{A}}$ & $0.98 \pm 0.14$ & $2.37 \pm 0.57$ & $1.76 \pm 0.63$ & 1.54 & $1.40 \pm$ & 0.27 & 0.04 & 0.20 \\
\hline \multicolumn{12}{|c|}{ Primary bile acids } \\
\hline CA total & $0.40 \pm 0.08$ & $0.32 \pm 0.12$ & $0.42 \pm 0.12$ & $0.23 \pm 0.03$ & $0.83 \pm 0.36$ & $0.58 \pm 0.37$ & $0.47 \pm 0.19$ & $0.40 \pm 0.16^{\mathrm{B}}$ & 0.25 & 0.06 & 0.43 \\
\hline $\begin{array}{c}\text { Inconjugated } \\
\text { CA }\end{array}$ & $0.22 \pm 0.06$ & $0.18 \pm 0.07$ & $0.21 \pm 0.09$ & $0.04 \pm 0.01$ & $0.65 \pm 0.35$ & $0.43 \pm 0.34$ & $0.20 \pm 0.15^{\mathrm{B}}$ & $0.20 \pm 0.16^{B}$ & 0.21 & $<0.01$ & 0.27 \\
\hline GCA & $0.16 \pm 0.04$ & $0.11 \pm 0.04$ & $0.10 \pm 0.01$ & $0.09 \pm 0.02$ & $0.16 \pm 0.05$ & $0.13 \pm 0.04$ & $0.13 \pm 0.04$ & $0.10 \pm 0.02$ & 0.94 & 0.22 & 0.94 \\
\hline CDCA total & $0.80 \pm 0.15$ & $0.59 \pm 0.16$ & $0.90 \pm 0.11^{\mathrm{A}}$ & $0.49 \pm 0.09^{B}$ & $0.96 \pm 0.21$ & $0.80 \pm 0.26$ & $0.70 \pm 0.19^{A}$ & $0.68 \pm 0.17$ & 0.61 & 0.15 & 0.08 \\
\hline $\begin{array}{l}\text { Unconjugated } \\
\text { CDCA }\end{array}$ & $0.36 \pm 0.07$ & $0.28 \pm 0.07$ & $0.36 \pm 0.11^{\mathrm{D}}$ & $0.12 \pm 0.03$ & $0.64 \pm 0.19$ & $0.43 \pm 0.20$ & $0.29 \pm 0.13^{c, D}$ & $0.31 \pm 0.16^{c}$ & 0.15 & $<0.001$ & 0.09 \\
\hline TCDCA & $0.15 \pm 0.04$ & $18 \pm 0.09$ & $0.08 \pm 0.01$ & $0.07 \pm 0.03^{B}$ & $0.15 \pm 0.07$ & $0.13 \pm 0.05$ & $0.08 \pm 0.02$ & $0.07 \pm 0.02$ & 0.48 & 0.16 & 0.90 \\
\hline GCDCA & $0.29 \pm 0.07$ & $0.13 \pm 0.03$ & $0.47 \pm 0.08$ & $0.29 \pm 0.08$ & $0.17 \pm 0.04$ & $0.25 \pm 0.10$ & $0.34 \pm 0.10$ & $0.30 \pm 0.08$ & 0.31 & 0.02 & 0.22 \\
\hline GDCA & $0.15 \pm 0.06$ & $0.16 \pm 0.09$ & $0.13 \pm 0.03$ & $0.08 \pm 0.03$ & $0.17 \pm 0.08$ & $0.12 \pm 0.05$ & $0.12 \pm 0.04$ & $0.08 \pm 0.02$ & 0.86 & 0.33 & 0.86 \\
\hline $\begin{array}{l}\text { LCA } \\
\text { unconjugated }\end{array}$ & $0.01 \pm 0.001$ & $0.02 \pm 0.005$ & $0.03 \pm 0.004^{E}$ & $0.02 \pm 0.003^{c}$ & $0.01 \pm 0.002$ & $0.02 \pm 0.003$ & $0.03 \pm 0.007^{\mathrm{E}}$ & $0.03 \pm 0.009^{\mathrm{E}}$ & 0.80 & $<0.001$ & 0.08 \\
\hline UDCA total & $0.15 \pm 0.04$ & $0.10 \pm 0.02$ & $0.10 \pm 0.03^{A}$ & $0.06 \pm 0.02$ & $0.27 \pm 0.09$ & $0.14 \pm 0.03^{B}$ & $0.08 \pm 0.02^{A, E}$ & $0.06 \pm 0.02^{\mathrm{E}}$ & 0.24 & $<0.001$ & 0.19 \\
\hline $\begin{array}{c}\text { Unconjugated } \\
\text { UDCA }\end{array}$ & $0.06 \pm 0.01$ & $0.06 \pm 0.01$ & $0.06 \pm 0.03^{A}$ & $0.04 \pm 0.02^{A}$ & $0.16 \pm 0.06$ & $0.09 \pm 0.03$ & $0.05 \pm 0.02^{\mathrm{A}, \mathrm{B}}$ & $0.05 \pm 0.02^{A, C}$ & 0.20 & 0.09 & 0.14 \\
\hline GUDCA & $0.09 \pm 0.029$ & $0.05 \pm 0.012$ & $0.03 \pm 0.008$ & $0.02 \pm 0.006^{B}$ & $0.12 \pm 0.045$ & $0.05 \pm 0.014^{B}$ & $0.02 \pm 0.005^{c}$ & $0.02 \pm 0.002^{c}$ & 0.57 & $<0.001$ & 0.75 \\
\hline \multicolumn{12}{|c|}{$\begin{array}{l}\text { Composition of plasma bile acids in response to exercise. Difference between time points (difference from before exercise) within modes of exercise: } \\
{ }^{B} P<0.05,{ }^{C} P<0.01,{ }^{E} P<0.001 \text { by } 2 \text {-way ANOVA with Dunnett (time) post hoc test for multiple comparisons. Difference between modes of exercise } \\
\text { for each time point: }{ }^{A} P<0.05 \text {, }{ }^{D} P<0.01 \text { by } 2 \text {-way ANOVA with Bonferroni (exercise) post hoc test for multiple comparisons. EE, endurance exercise; } \\
\text { RE, resistance exercise; E, Exercise; T, Time; TBA, total bile acids; CA, cholic acid; TCA, taurocholic acid; GCA, glycocholic acid; CDCA, chenodeoxycholic } \\
\text { acid; TCDCA, taurochenodeoxycholic acid; GCDCA, glycochenodeoxycholic acid; DCA, deoxycholic acid; GDCA, glycodeoxycholic acid; LCA, lithocholic } \\
\text { acid; UDCA, ursodeoxycholic acid; GUDCA, glycoursodeoxycholic acid. } P \text { values shown in bold are significant. }\end{array}$} \\
\hline
\end{tabular}

Primary BAs. There was an effect of time on unconjugated cholic acid (CA) and chenodeoxycholic acid (CDCA) $(P<0.01$ and $P<0.001$, respectively; Table 1$)$, and both decreased 60 and 180 minutes after RE (both time points, $P<0.05$ for CA and $P<0.01$ for CDCA; Table 1). Conversely, taurocholic acid (TCA) showed an effect of time $(P<0.001)$ and increased 60 minutes after both EE and RE $(P<0.05$ and $P<$ 0.01 , respectively; Table 1$)$. Glycochenodeoxycholic acid (GCDCA) showed effect of time $(P=0.02)$, but the concentrations were not statistically different from concentrations before exercise for any exercise mode (Table 1). The concentrations of plasma glycocholic acid (GCA) and taurochenodeoxycholic acid (TCDCA) were unchanged following both EE and RE, except for a small decrease in TCDCA at 180 minutes after EE compared with concentrations before exercise $(P=0.04$; Table 1$)$.

Secondary BAs. The unconjugated deoxycholic acid (DCA) plasma concentration increased $(P<0.05) 60$ minutes after EE compared with the concentration before exercise and showed a significant time effect $(P<$ 0.01; Table 1). Glycodeoxycholic acid (GDCA) was unaffected by both modes of exercise. Unconjugated lithocholic acid (LCA) showed a time effect $(P<0.001)$ and was elevated 60 and 180 minutes following both modes of exercise $(P<0.001$ and $P<0.05$ for EE; $P<0.01$ and $P<0.001$ for RE; Table 1$)$. Total ursodeoxycholic acid (UDCA) showed a time effect $(P<0.001$; Table 1$)$ and was reduced immediately after $\mathrm{RE}(P<0.05)$ and decreased further 60 and 180 minutes after exercise $(P<0.001$ for both time points). Unconjugated UDCA plasma concentration decreased at 60 and 180 minutes after RE compared with the concentration before exercise $(P<0.05$ and $P<0.01$, respectively; Table 1$)$. There was an effect of time on glycoursodeoxycholic acid (GUDCA) $(P<0.001)$, which decreased immediately following RE $(P<0.05)$ and showed a further gradual decrease at the 60 - and 180 -minute time points $(P<0.01$ for both time points; Table 1$)$. Following EE, GUDCA showed a similar pattern that reached statistical significance 180 minutes after exercise $(P<0.05$; Table 1$)$. 


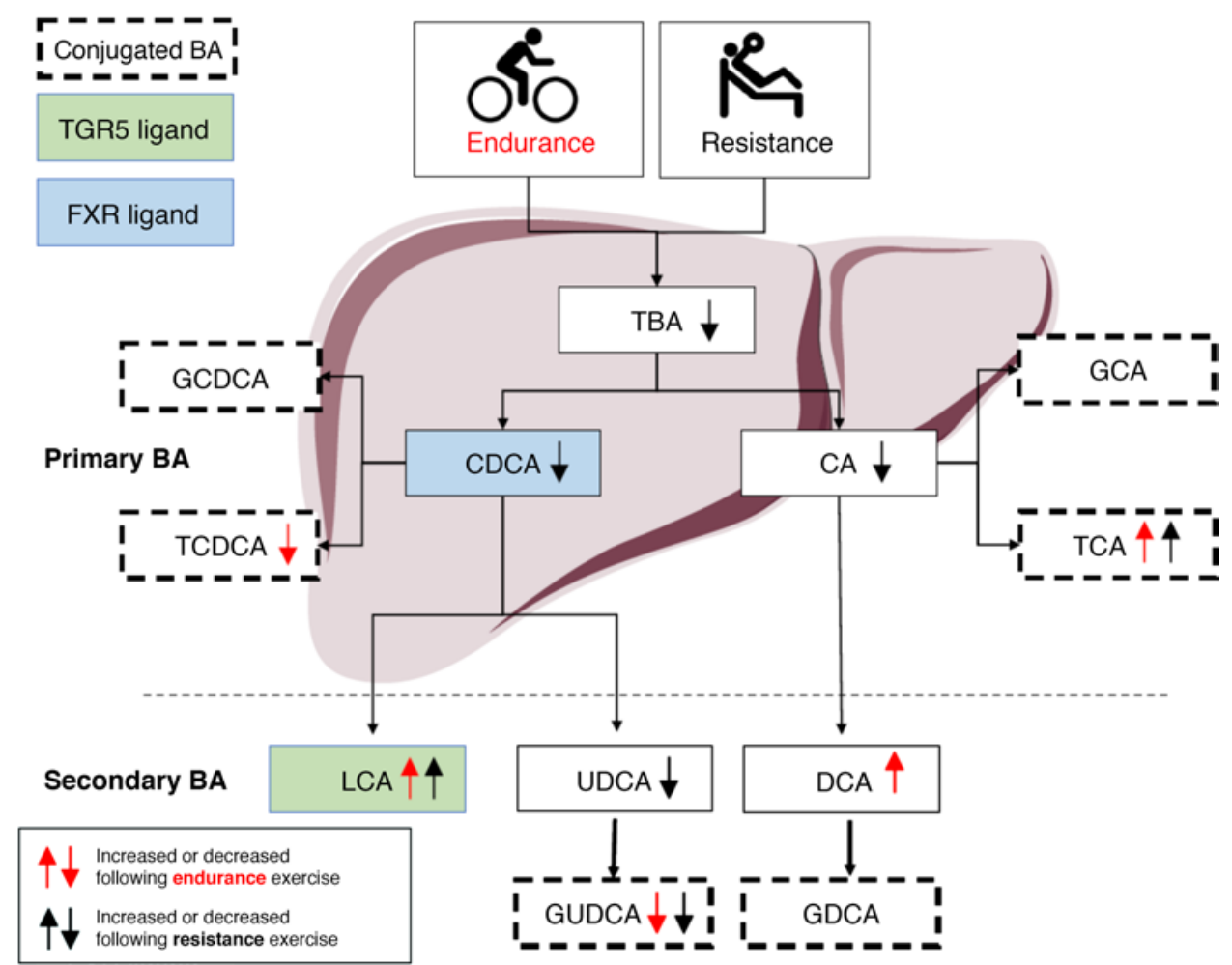

Figure 4. Overview of exercise mode-governed regulation of plasma bile acid composition. Plasma TBA and primary bile acids CA and CDCA were decreased with resistance exercise. The primary bile acid, TCA, increased independent of exercise mode. Primary bile acid TCDCA decreased with endurance exercise. The secondary bile acid and potent TCR5 agonist LCA (highlighted in green) circulated at very low plasma concentrations at rest and highly increased with both modes of exercise. Secondary bile acid GUDCA was decreased with both modes of exercise. UDCA was decreased with resistance exercise, and DCA was increased with endurance exercise. BA, bile acid; TBA, total bile acids; CDCA, chenodeoxycholic acid; CA, cholic acid; LCA, lithocholic acid; UDCA, ursodeoxycholic acid; DCA, deoxycholic acid; GUDCA, glycoursodeoxycholic acid; GDCA, glycodeoxycholic acid; GCA, glycocholic acid; TCA, taurocholic acid; GCDCA, glycochenodeoxycholic acid; TCDCA, taurochenodeoxycholic acid; TCR5, takeda G protein-coupled receptor 5 ; FXR, farnesoid $X$ receptor.

Time-resolved coregulation of BA/FGF19 and glucagon/FGF21 is exercise mode dependent To understand if exercise mode-linked alterations in FGF19 and FGF21 couple to their respective upstream endocrine secretagogues, we analyzed relative coordinated changes in TBA/FGF19 and in glucagon/ FGF21 (Figure 5). Whereas EE seems to temporally uncouple the TBA/FGF19 axis, we observed a time-dependent coordinated decrease in the TBA and FGF19 in response to RE (Figure 5, A and B). A decrease in plasma TBA $(P<0.05$ at 120 minutes) preceded a subsequent decrease in plasma FGF19 $(P<0.01$ at 240 minutes) (Figure 5B). A robust induction in glucagon immediately following $\mathrm{EE}(P<0.001$ relative to before exercise) was followed by a corresponding pronounced induction in plasma FGF21 60 minutes later $(P<$ $0.001,90-150$ minutes relative to before exercise) (Figure 5C). In contrast, RE did not engage the glucagon/ FGF21 axis (Figure 5D). In summary, these analyses unravel the exercise mode-dependent activity of endocrine circuits that lead to the release of circulating FGF19 and FGF21, respectively.

\section{Discussion}

Here, we report, for the first time to our knowledge, effects of exercise on plasma concentrations of BA species, FGF19, and FGF21 in healthy humans. We observed a coordinated decrease in TBA and FGF19 in the recovery period following high-volume RE. Conversely, we found that plasma FGF21 was increased with EE and that a robust increase in plasma glucagon precedes the FGF21 increase, suggesting a coordinated regulation of glucagon and FGF21 with EE. Notably, while most BA species were decreased with exercise, we observed a high induction in the TGR5 receptor agonist, LCA, with both EE and RE. In summary, our work emphasizes that distinct modes of exercise uniquely affect plasma concentrations of individual BAs, FGF19, and FGF21 in healthy humans, suggesting that these alterations may underlie some of the metabolic benefits of exercise. 
TBA - FGF19

A

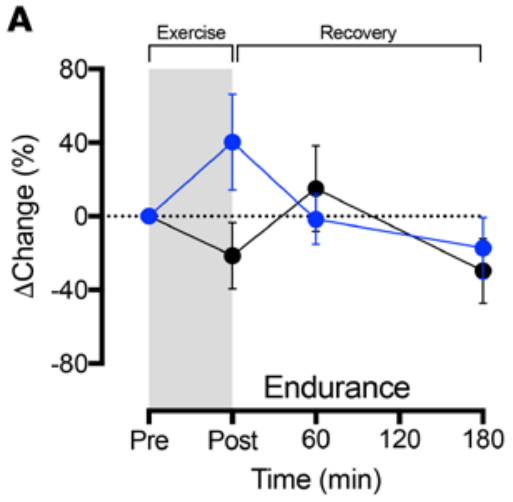

B

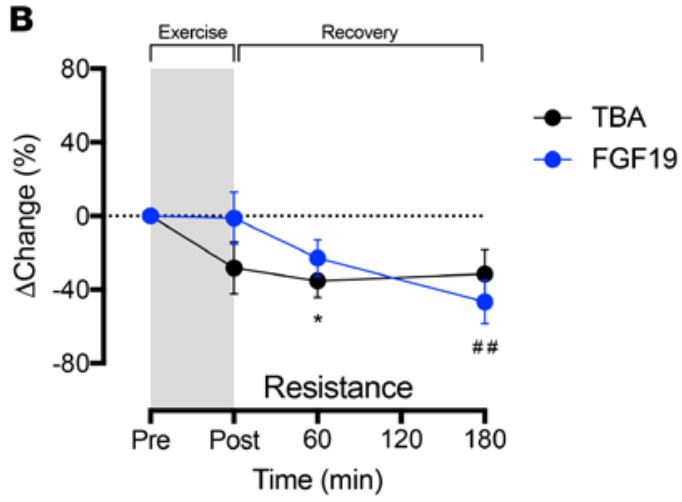

Glucagon - FGF21

C

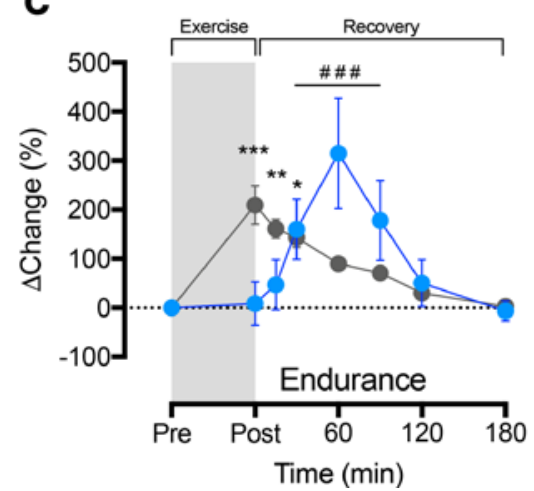

D

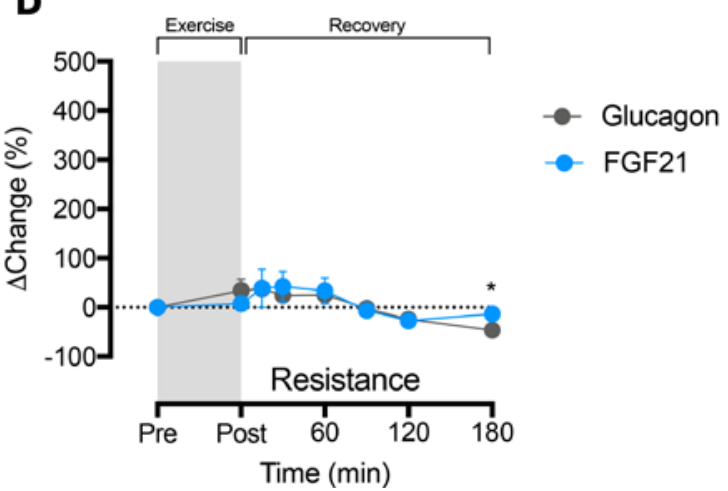

Figure 5. Comparison of coregulated TBA/FGF19 and glucagon/FGF21 in response to exercise mode. Concentrations of total bile acids (TBA; black, $n=10$ for each exercise mode) and FGF19 (blue, $n=10$ for each exercise mode) are shown as $\Delta$ change (\%) for endurance exercise (A) and resistance exercise (B). Concentrations of plasma glucagon (dark gray, $n$ $=10$ for each exercise mode) and FGF21 (light blue, $n=8$ for each exercise mode) are shown as $\Delta$ change (\%) for endurance exercise (C) and resistance exercise (D). Data are presented as mean \pm SEM. Difference between time points (difference from before exercise) by 2-way ANOVA with Dunnett post hoc test for multiple comparisons. For TBA and glucagon: ${ }^{*} P<0.05,{ }^{* *} P<0.01,{ }^{* *} P<0.001$. For FGF19 and FGF21: ${ }^{\# \#} P<0.01,{ }^{\# \# \# P<0.001 .}$

BAs are increasingly acknowledged as signaling molecules with immunometabolic health benefits (2). These versatile molecules exert important endocrine and paracrine functions apart from their role as digestive surfactants. Thus, BAs are involved in the regulation of lipid metabolism, glycemic control, and energy expenditure and have been implicated in the metabolic benefits of both bariatric surgery (15) and cold exposure (16). Here, we link exercise to BA metabolism and unravel how distinct BA species are regulated in response to $\mathrm{RE}$ and $\mathrm{EE}$, respectively. We found that the most potent natural TGR5 receptor agonist, LCA, is increased approximately 2.5 -fold following both modes of exercise. The TGR5 receptor is believed to be ubiquitously expressed in humans, and convergent findings suggest pleiotropic effects of TGR5 receptor activation, including induction of brown adipose tissue thermogenesis (4), lowering of proinflammatory cytokines (17), and improved glucose tolerance (5), all of which are hallmark outcomes following exercise. However, with $\mathrm{EC}_{50}$ values 10 -fold higher than the concentrations of LCA in human plasma (18), it is questionable whether the exercise-induced increase in LCA has any relevant signaling capacity in metabolic target tissues, such as adipose tissue and skeletal muscle. Conversely, basolateral TGR5 receptors may be exposed to higher concentrations of BAs than that detected in venous blood, suggesting that exercise-induced induction in LCA locally could affect enteroendocrine cellular signaling. 
A central finding of the present study is that TBAs were decreased in the recovery period following RE. In comparison, a recent study reported that a half-marathon run lowers TBAs in middle-aged men and women (19). The running-induced decrease in TBAs was reflected in lower concentrations of CA, DCA, CDCA, and UDCA. In contrast, we found that bicycling EE only has subtle effects of circulating BAs but that RE reduces circulating concentrations of the primary BA CA and the unconjugated form of CDCA. This discrepancy may reflect that Danese et al. measured BA in a diverse group of subjects, with mixed sex, age, and training status, without controlling for exercise intensity or energy intake (19). Here, we measured BA composition under controlled fasting conditions and at a high time resolution after exercise. With our experimental design, we observed that many BAs are significantly altered in the time period 1-3 hours following an exercise bout and that exercise mode plays a significant role to this regulation.

The growing interest in BAs as metabolic signaling molecules may be partially ascribed to the fact that the endogenous FGF19 is expressed in response to BA-induced activation of FXR. Notably, FGF19 acts in the brain to decrease glucose concentrations and increase energy expenditure (20,21). More recently, pharmacological FGF19 was found to have anabolic effects on skeletal muscle in mice (9), prompting us to hypothesize that a enterohepatic muscle axis might implicate that FGF19 promotes muscle growth in response to resistance training. However, here, we present evidence opposing this hypothesis. Instead, RE potently decreases circulating FGF19 in the recovery period. This alludes to the possibility of the BA/FGF19 axis being involved in the physiological adaptations to resistance training and that suppression, rather than an induction of the axis, could mediate such adaptations. Future studies are required to explore if concurrent increases in, for example, insulin and glucose in conjunction with the suppression of the BA/FGF19 axis following RE are intertwined.

In agreement with a growing number of studies, we found that EE increases FGF21 (10-14). Here, we show that FGF21 induction mirrors that of glucagon, with a 1-hour delay between their respective peak levels, supporting the idea of a glucagon/FGF21 axis (22) that is regulated by EE. Surprisingly, high-volume RE had no effect on glucagon or FGF21. Thus, contraction-induced metabolic stress, evident by the considerable increase in plasma lactate following RE, dissociates from the metabolic pathways, leading to elevated FGF21 following EE. Supporting the connection between FGF21 and glucagon, the glucagon concentrations following RE were unchanged and gradually declined during the recovery period.

Certain limitations to our study should be highlighted. The endocrine factors examined herein are all regulated by feeding status and/or by specific macronutrients. Thus, to limit confounding variables, we examined the exercise and postexercise effects under fasting conditions, following controlled dietary intake on the nights before test days. A previous study found that prolonged fasting gradually decreases BA and FGF19 in circulation (23), thus we cannot exclude that the suppression of the TBA/FGF19 axis following $\mathrm{RE}$ is partially amplified by prolonged fasting. Conversely, because we found distinct RE and EE endocrine signatures, we believe that our results are indicative of exercise-specific physiological regulations and are not the consequence of prolonged fasting. This notion is supported by the fact that several BA subspecies were increased by exercise. Another limitation is that our findings only apply to young relatively welltrained men. Thus, future studies should map out potential differences in exercise-induced alterations to plasma BA composition and circulating FGF19 and FGF21 in relation to sex, age, and metabolic status. Finally, a single bout of exercise provides limited information. Training programs for both EE and RE and genetic and pharmacological animal studies are needed to gauge causality of the herein reported alterations in plasma BA, FGF19, and FGF21 and a possible implication for exercise-induced health benefits.

In conclusion, we report distinct effects of $\mathrm{EE}$ and $\mathrm{RE}$ on circulating concentrations of BA species, FGF19, and FGF21 in young healthy humans. For the first time to our knowledge, we identify temporal relationships between decreased BA and FGF19 following resistance training and a sharp disparity in FGF21 concentrations with EE eliciting a clear increase parallel to that of glucagon.

\section{Methods}

\section{Subjects and study design}

Ten recreationally active male subjects participated in the study. Because plasma BAs are reported to fluctuate with the menstrual cycle (24), we decided to recruit exclusively male subjects for this investigation. Participants were healthy and did not use any medication or ergogenic supplements (age, $24 \pm 1$ years; weight, $80.6 \pm 2.1 \mathrm{~kg}$; body mass index, $23.7 \pm 0.6 \mathrm{~kg} / \mathrm{m}^{2}$; fat percentage, $12.7 \% \pm 1.0 \%$; peak oxygen uptake $\left[\mathrm{VO}_{2 \text { peak }}\right], 4.48 \pm 0.171 / \mathrm{min}[55.7 \pm 1.7 \mathrm{ml} / \mathrm{min} / \mathrm{kg}][$ mean $\left.\pm \mathrm{SEM}]\right)$. 
The study design is shown in Figure 1. Subjects reported to our laboratory on 3 different days. On the first day, 10 repetition maximum (RM) was determined for the REs (described in detail below), and $\mathrm{VO}_{\text {2peak }}$ was determined using an Online system (Oxycon Pro system, Jaeger) and an incremental ergometer bike test (Monark, 828E). Values for $\mathrm{VO}_{2 \text { peak }}$ were accepted when oxygen uptake was leveling off or decreased with increasing work load and respiratory exchange ratio exceeded 1.15. On the second and third test day, the subjects were overnight fasted for at least 10 hours, and they abstained from vigorous physical activity the day before. The subjects were asked to record their meals the day before the second test and to consume similar meals the night before the third test. The two experimental days were separated by 6-12 days. Body composition was determined with a dual energy x-ray absorptiometry scanner (Lunar iDXA, G\&E Medical Systems) on the second test day. The subjects had access to ad libitum water throughout the test days.

\section{Experimental procedures}

A peripheral venous catheter was placed in the cephalic vein for the RE experiment or the median cubital vein for the EE experiment. After a minimum of 10 minutes of supine rest, a basal blood sample was drawn. Subjects were randomized to perform a 1-hour exercise session of either RE or EE. Immediately after exercise a blood sample was drawn, and the subject was placed in a supine position for the remaining time. Blood samples were then drawn at the following times after termination of exercise: 15, 30, 60, 90, 120, and 180 minutes. Apart from the resting sample and the sample immediately after exercise BAs were only measured at 60 and 180 minutes after exercise.

\section{Exercise protocol}

To investigate how distinct modes of exercise, EE versus RE, effect BAs, FGF19, and FGF21, 1 hour of characteristic exercise protocols aiming to (a) maximize the strain on the cardiovascular system with $\mathrm{EE}$ and (b) promote the greatest hypertrophy response with RE were used. Both exercise programs were designed to match the moderately trained status of the subjects, with high-intensity cycling for EE and high-volume weight training for RE.

$R E$. Prior to RE, the subjects warmed up with 5 minutes of cycling at 100 watts and 15 repetitions of unweighted squats, push-ups, sit-ups, and back raises. The RE protocol consisted of 5 resistance drills performed in the order mentioned: isolated knee extension, barbell bench press, seated hamstring curls, wide-grip lateral pulldown, and leg press. The subjects performed 5 sets of 10 repetitions with 90 seconds of rest between sets in accordance with the guidelines from the American College of Sports Medicine for trained adults (25). In keeping with the guidelines, this high-volume exercise protocol promotes local metabolic and systemic hormonal stress and is recognized to promote skeletal muscle hypertrophy. The subjects were instructed to apply a controlled eccentric phase. The starting load was set to $90 \%-95 \%$ of $10 \mathrm{RM}$, which resulted in positive failure within the first 3-4 sets. The load was adjusted accordingly, aiming to ensure that the subject could complete at least 8 repetitions in the last set. The total time of RE, including warm-up, was 58-59 minutes for all subjects. One subject did not perform the fifth and last leg press set due to nausea.

$E E$. The 60 minutes of EE was performed on an ergometer bike (Monark). The first 10 minutes, a warm-up period, was split between 100 watts for 5 minutes and 150 watts for 5 minutes. The remaining 50 minutes of $\mathrm{EE}$ was done at an individual workload calculated to elicit $70 \% \mathrm{VO}_{2 \text { peak }}$, aiming at the highest feasible workload that moderately trained subjects can sustain for 1 hour. After 5 minutes of this workload oxygen uptake was measured (Oxycon Pro system, Jaeger), and small adjustments to workload were made for some subjects in order to achieve the desired work-out intensity. Oxygen uptake was measured 2-3 more times throughout the 60 minutes of bike exercise, and additional changes in workload were made for a few subjects. The average oxygen uptake during the 50 minutes of cycling at the individual workload was $74 \%$ of $\mathrm{VO}_{2 \text { peak. }}$ All subjects completed the full 60 minutes of cycling.

\section{Blood samples and plasma analyses}

Blood samples were centrifuged at $3,756 \mathrm{~g}$ and $4^{\circ} \mathrm{C}$ for 10 minutes, and the supernatant was kept at $-80^{\circ} \mathrm{C}$ until analysis. Glucose and lactate in whole blood were analyzed immediately after the blood samples were drawn (ABL Flex 830, Radiometer). Plasma FGF19 (R\&D Systems, DF1900), FGF21 (R\&D Systems, DF2100), glucagon (Mercodia, 10-1271-01), and insulin (Roche, COBAS 6000) were analyzed using commercially available ELISA kits in accordance with the manufacturer's instructions. 


\section{LC-MS BA quantitation in bile}

Chemicals. All BA standards were purchased from either MilliporeSigma or Steraloids. Liquid chromatography-mass spectrometry-grade (LC-MS-grade) water, acetonitrile, methanol, 2-propanol, formic acid, and all other reagents were purchased from MilliporeSigma unless otherwise stated.

Sample preparation and $L C$. Samples were thawed on wet ice. $150 \mu 1$ EDTA plasma was combined with $10 \mu \mathrm{l} 1.2 \mu \mathrm{M}$ internal standard solution (taurolithocholic acid- $\mathrm{d}_{4}$ [TLCA- $\mathrm{d}_{4}$ ], taurodeoxycholic acid- $\mathrm{d}_{4}$ [TDCA- $\mathrm{d}_{4}$ ], DCA-d $\mathrm{d}_{6}$, CA- $\mathrm{d}_{5}$, TCA- $\mathrm{d}_{4}$, tauroursodeoxycholic acid- $\mathrm{d}_{4}$ [TUDCA-d $\mathrm{d}_{4}$, LCA- $\mathrm{d}_{5}$, and CDCA- $\mathrm{d}_{4}$ ). $525 \mu 1$ ice-cold LC-MS-grade methanol was then added to plasma. Samples were immediately vortexed $(\sim 3 \mathrm{~s}$ at $20,000 \mathrm{rpm})$ and then placed back on wet ice. Supernatants were clarified with centrifugation $(20,238 \mathrm{~g}, 10$ minutes, ambient temperature). Supernatants were dried via speed vacuum and then stored at $-80^{\circ} \mathrm{C}$. Dried samples were resuspended in $50 \mu 195 / 5 \%$ mobile phase A/B (see below) immediately before analysis. Extracts were clarified with centrifugation (same as above). $45 \mu 1$ supernatant was transferred to a fresh Verex vial (catalog AR0-3920-12, Phenomenex ApS). A pooled quality control (QC) sample was created by combining equal volumes $(5 \mu \mathrm{l})$ of each sample. These vials were capped Verex Cert+MSQ PTFE cap (catalog AR0-8952-12-M, Phenomenex ApS). Samples were randomized and placed in a Dionex Ultimate 3000 autosampler without temperature control. Analytes were injected $(15 \mu \mathrm{l})$ onto a heated $\left(40^{\circ} \mathrm{C}\right)$ Phenomenex PS C18 column $(2.1 \times 100 \mathrm{~mm}, 1.7 \mu \mathrm{m})$ and separated at a flow rate of $0.4 \mathrm{ml} / \mathrm{min}$ using a gradient between mobile phase A $(5 \mathrm{mM}$ ammonium acetate with $0.1 \%$ [v/v] formic acid) and $\mathrm{B}(0.1 \%$ $[\mathrm{v} / \mathrm{v}]$ formic acid in acetonitrile). The gradient consisted of a hold at $5 \% \mathrm{~B}$ for 1 minute, followed by a linear ramp to $95 \%$ B over 9 minutes. The column was then washed for 5 minutes at $95 \%$ B and reequilibrated for 5 minutes at $5 \% \mathrm{~B}$.

Mass spectrometry. Before beginning analysis, the instrument was calibrated by directly infusing sodium formate. Calibration was modeled with HPC mode. Calibrant was also infused at the beginning of each analysis. A lock mass (Hexakis [1H, 1H, 2H-perfluoroethoxy] phosphazene in 2-propanol [0.1 mg/ml]) was employed to improve mass accuracy over the duration of analysis. Analytes ionized via electrospray were detected in full scan $(m / z: 50-1,000)$, negative-ion mode with a Bruker Impact II Q-TOF. The instrument was operated using the following conditions: cone voltage, $30 \mathrm{kV}$; collision energy, $30 \mathrm{eV}$; nebulizer gas flow, $101 / \mathrm{min}$; nebulizer gas temperature, $220^{\circ} \mathrm{C}$; nebulizer pressure, $2 \mathrm{bar}$; and scan rate, $3 \mathrm{~Hz}$.

Quantitation. BAs were quantified using an internally controlled standard curve, with calibration points at the following final concentrations in a $50-\mu 1$ volume: $0.03,0.06,0.1,0.3,0.6,1,2,3$, and $5 \mu \mathrm{M}$. The BAs detected and quantified are as follows: CA, CDCA, DCA, GCA, GCDCA, GDCA, GUDCA, LCA, muricholic acid $\alpha$ (MCA $\alpha$ ), MCA $\beta$, MCA $\gamma$, TCA, TDCA, tauromuricholic acid $\alpha$ (TMCA $\alpha$ ), TMCA $\beta$, TMCA $\gamma$, TLCA, TUDCA, and UDCA. The pooled QC was injected in triplicate at the beginning of the queue. In subsequent injections, the pooled $\mathrm{QC}$ was injected singly when half the samples were injected and at the end of the queue. Standard QCs were produced singly at concentrations of $0.25,2.5$, and 12.5 $\mu \mathrm{M}$. Standard QCs were injected after the QC pooled injections in the beginning, middle, and end of the sequence. Plasma from an unrelated cohort were dosed with internal standard and either water or the $0.25,2.5$, or $12.5 \mu \mathrm{M} \mathrm{QC}$ and injected at the beginning and end of the sequence. For all BAs but CDCA, molar amounts were determined using a standard curve, whereby the concentration versus the ratio of the external standard to the internal standard was plotted. CDCA molar values were determined through comparison to the CDCA- $\mathrm{d}_{4}$ area within the sample. Final values for all BAs were determined by correcting for dilution factor. Retention times and $m / z$ ratios for analytes and internal standard are included in Supplemental Table 1 (supplemental material available online with this article; https://doi.org/10.1172/jci. insight.122737DS1). The internal standard used for quantification is also indicated in Supplemental Table 1. Values below quantification limit $(\mathrm{QL} \leq 0.01 \mu \mathrm{M})$ were predefined as/set at $0.01 \mu \mathrm{M}$. Species for which $>50 \%$ of the values at all time points were below the $\mathrm{QL}$ were excluded from the analysis.

\section{Statistics}

All statistical analyses were performed using GraphPad Prism 7.0. Two-way ANOVA with repeated measures was used to test for both within-group (time) and between-group (exercise) differences for parameters measured during both types of exercise. For analyses of blood glucose and lactate as well as plasma insulin and glucagon between-group differences were analyzed. For all other parameters, both within-group as well as between-group differences were analyzed. Using incremental values for between-group analysis allowed us to compare responses without the potential confounding effect of a difference in the fasting concentrations. 
Whenever the ANOVA tests revealed significant effects, the Bonferroni (exercise) or the Dunnett (time) post hoc test for multiple comparisons was used. $P<0.05$ was considered significant. Data that were not normally distributed or had unequal variance at baseline were log transformed before statistical analyses. Data are presented as mean \pm SEM.

\section{Study approval}

The subjects were informed about the possible risks and discomfort involved before written consent to participate was obtained. The study was approved by the local ethics committee of Copenhagen and Frederiksberg (H-17030450), and all procedures were carried out in accordance to the Declaration of Helsinki.

\section{Author contributions}

TM researched and interpreted the data and wrote the manuscript. RES researched and interpreted the data and cowrote the manuscript. SAJT performed the LC-MS analysis, interpreted the data, and edited the manuscript. JSS assisted with the LC-MS analysis. MPG and JWH interpreted the data and edited the manuscript. TM and CC conceptualized the project. CC researched and interpreted the data and cowrote the manuscript. CC is the guarantor of the work, had full access to all the data, and takes responsibility for the integrity of the publication.

\section{Acknowledgments}

We thank Charlotte Sashi Aier Svendsen and Jeppe Bach for technical support. CC receives funding from the Lundbeck Foundation (fellowship R238-2016-2859) and the Novo Nordisk Foundation (grant NNF17OC0026114). The Novo Nordisk Foundation Center for Basic Metabolic Research is an independent Research Center, based at the University of Copenhagen, Denmark, and partially funded by an unconditional donation from the Novo Nordisk Foundation.

Address correspondence to: Christoffer Clemmensen, University of Copenhagen, Blegdamsvej 3B, 2200, Copenhagen, Denmark. Phone: 45.22916333; Email: chc@sund.ku.dk.

1. Zierath JR, Wallberg-Henriksson H. Looking ahead perspective: where will the future of exercise biology take us? Cell Metab. 2015;22(1):25-30.

2. Chávez-Talavera O, Tailleux A, Lefebvre P, Staels B. Bile acid control of metabolism and inflammation in obesity, type 2 diabetes, dyslipidemia, and nonalcoholic fatty liver disease. Gastroenterology. 2017;152(7):1679-1694.e3.

3. Duboc H, Taché Y, Hofmann AF. The bile acid TGR5 membrane receptor: from basic research to clinical application. Dig Liver Dis. 2014;46(4):302-312.

4. Broeders EP, et al. The bile acid chenodeoxycholic acid increases human brown adipose tissue activity. Cell Metab. 2015;22(3):418-426.

5. Thomas C, et al. TGR5-mediated bile acid sensing controls glucose homeostasis. Cell Metab. 2009;10(3):167-177.

6. Inagaki T, et al. Fibroblast growth factor 15 functions as an enterohepatic signal to regulate bile acid homeostasis. Cell Metab. 2005;2(4):217-225.

7. Beenken A, Mohammadi M. The FGF family: biology, pathophysiology and therapy. Nat Rev Drug Discov. 2009;8(3):235-253.

8. Owen BM, Mangelsdorf DJ, Kliewer SA. Tissue-specific actions of the metabolic hormones FGF15/19 and FGF21. Trends Endocrinol Metab. 2015;26(1):22-29.

9. Benoit B, et al. Fibroblast growth factor 19 regulates skeletal muscle mass and ameliorates muscle wasting in mice. Nat Med. 2017;23(8):990-996.

10. Kim KH, Kim SH, Min YK, Yang HM, Lee JB, Lee MS. Acute exercise induces FGF21 expression in mice and in healthy humans. PLoS ONE. 2013;8(5):e63517.

11. Tanimura Y, et al. Acute exercise increases fibroblast growth factor 21 in metabolic organs and circulation. Physiol Rep. 2016;4(12):e12828.

12. Hansen JS, Pedersen BK, Xu G, Lehmann R, Weigert C, Plomgaard P. Exercise-induced secretion of FGF21 and follistatin are blocked by pancreatic clamp and impaired in type 2 diabetes. J Clin Endocrinol Metab. 2016;101(7):2816-2825.

13. Cuevas-Ramos D, et al. Exercise increases serum fibroblast growth factor 21 (FGF21) levels. PLoS ONE. 2012;7(5):e38022.

14. Loyd C, et al. Fibroblast growth factor 21 is required for beneficial effects of exercise during chronic high-fat feeding. $J A p p l$ Physiol. 2016;121(3):687-698.

15. Ryan KK, et al. FXR is a molecular target for the effects of vertical sleeve gastrectomy. Nature. 2014;509(7499):183-188.

16. Worthmann A, et al. Cold-induced conversion of cholesterol to bile acids in mice shapes the gut microbiome and promotes adaptive thermogenesis. Nat Med. 2017;23(7):839-849.

17. Yoneno K, et al. TGR5 signalling inhibits the production of pro-inflammatory cytokines by in vitro differentiated inflammatory and intestinal macrophages in Crohn's disease. Immunology. 2013;139(1):19-29.

18. Kawamata Y, et al. A G protein-coupled receptor responsive to bile acids. J Biol Chem. 2003;278(11):9435-9440. 
19. Danese E, Salvagno GL, Negrini D, Brocco G, Montagnana M, Lippi G. Analytical evaluation of three enzymatic assays for measuring total bile acids in plasma using a fully-automated clinical chemistry platform. PLoS One. 2017;12(6):e 0179200.

20. Ryan KK, Kohli R, Gutierrez-Aguilar R, Gaitonde SG, Woods SC, Seeley RJ. Fibroblast growth factor-19 action in the brain reduces food intake and body weight and improves glucose tolerance in male rats. Endocrinology. 2013;154(1):9-15.

21. Morton GJ, et al. FGF19 action in the brain induces insulin-independent glucose lowering. J Clin Invest. 2013;123(11):4799-4808.

22. Habegger KM, et al. Fibroblast growth factor 21 mediates specific glucagon actions. Diabetes. 2013;62(5):1453-1463.

23. Lundåsen T, Gälman C, Angelin B, Rudling M. Circulating intestinal fibroblast growth factor 19 has a pronounced diurnal variation and modulates hepatic bile acid synthesis in man. J Intern Med. 2006;260(6):530-536.

24. Pennington CR, Ross PE, Murison J, Bouchier IA. Fluctuations of serum bile acid concentrations during the menstrual cycle. J Clin Pathol. 1981;34(2):185-186.

25. American College of Sports Medicine. American College of Sports Medicine position stand. Progression models in resistance training for healthy adults. Med Sci Sports Exerc. 2009;41(3):687-708. 